\title{
Desmoplastic malignant mesothelioma: a review of 17 cases
}

\author{
G E Wilson, P S Hasleton, A K Chatterjee
}

\author{
Abstract \\ Aims: To identify the histological \\ features of desmoplastic mesothelioma, \\ and to determine its incidence and prog- \\ nosis.
}

Methods: Two hundred and fifty five cases of malignant mesothelioma were examined over seven years (1982-9) to identify the desmoplastic variant. Sections were cut at $5 \mu \mathrm{m}$ and stained with haemotoxylin and eosin and with CAM $5 \cdot 2$ (Dakopatts). Asbestos fibre counts were carried out by light microscopy in 14 cases using the potash digestion method.

Results: Seventeen cases were identified as desmoplastic mesothelioma giving an incidence of $6.6 \%$. In 11 cases the cell type in more cellular areas was sarcomatous and in six others it was biphasic. The mean survival time from onset of symptoms to death was $5 \cdot 8$ months for the sarcomatous variant and 6.8 months for the biphasic variant. Twelve of 16 patients had had previous occupational exposure to asbestos, ranging from five months to 43 years. The diagnosis of desmoplastic mesothelioma was only accepted if acellular connective tissue comprised $50 \%$ or more of the tumour bulk. Also seen was collagen necrosis, anastomosing bands of often hyalinised collagen with a prominent storiform pattern, and where cellular detail was present there were hyperchromatic nuclei.

Conclusions: Desmoplastic mesothelioma is a rare variant of malignant mesothelioma with a storiform collagen pattern, collagen necrosis, bland acellular collagen and focal cytological features of malignancy. Though rare, it is important to recognise this variant and distinguish it from a pleural plaque, nonspecific reactive pleural fibrosis, pleurisy, rheumatoid disease, or, rarely, spindle cell sarcomas.

Department of Pathology, Regional Cardiothoracic Centre, Wythenshawe Hospital, Manchester M23 9LT

GE Wilson

P S Hasleton

Medical Boarding

Centre (Respiratory

Diseases), Manchester

A K Chatterjee

Correspondence to:

Dr P S Hasleton.

Accepted for publication

23 September 1991 years for the effects of exposure to asbestos to develop into mesothelioma, it is expected that the number of cases will continue to increase until 2000 and beyond. ${ }^{124}$
There is a subgroup of malignant mesothelioma, termed desmoplastic malignant mesothelioma (DMM), in which $50 \%$ or more of the tumour is fibrous and has a low cell content. ${ }^{67}$ The hypocellular, bland appearances of such desmoplastic areas can be extremely difficult to interpretate and the correct diagnosis of malignancy may not be suspected. This is particularly the case when needle biopsy is performed, and many cases of DMM are still only diagnosed at necropsy.

\section{Methods}

Two hundred and fifty five cases of malignant mesothelioma were seen at or referred to the regional cardiothoracic centre between 1982 and 1989.

Seventeen $(6.6 \%)$ of these cases were considered to be DMM-that is, more than $50 \%$ of the tumour was composed of dense hypocellular fibrous tissue. In seven patients the diagnosis was made during life by thoracotomy and biopsy. Post mortem examinations were carried out in 16 of the 17 cases. Clinical and occupational histories were taken from hospital records or from the Medical Boarding Panel (Respiratory Diseases) notes. Relevant blocks of tissue were fixed in formalin and embedded in paraffin wax. Sections $(5 \mu \mathrm{m})$ were stained with haematoxylin and eosin for light microscopical examination. An average of seven blocks of tumour per case were available for study (range two to 19). Immunohistochemical staining was done on formalin fixed tissue using the standard peroxidase method $^{8}$ followed by application of the low molecular weight cytokeratin antibody CAM 5.2 (Dakopatts). Counts of asbestos fibres were carried out by light microscopy in 14 cases using the potash digestion method. ${ }^{9}$

The ages of 16 of the 17 patients were known and ranged from 41-79 years (mean $60 \cdot 1$ years) at the time of diagnosis. Fifteen patients were male and two female. In 11 the presenting complaints included pleural effusion in five, shortness of breath in three, and anaemia, pleuritic chest pain, and weight loss, respectively in three. The length of survival from onset of symptoms was known in 12 cases, the mean survival being 5.8 months in eight patients with sarcomatous tumours and $6 \cdot 8$ months in four patients with mixed tumours.

Twelve of 16 patients had had previous occupational exposure to asbestos. The duration of exposure ranged from five months to 43 years where it was known. Specific treatment was given in five cases and included pleural decortication in three, radiation in one, and chemotherapy in one. 


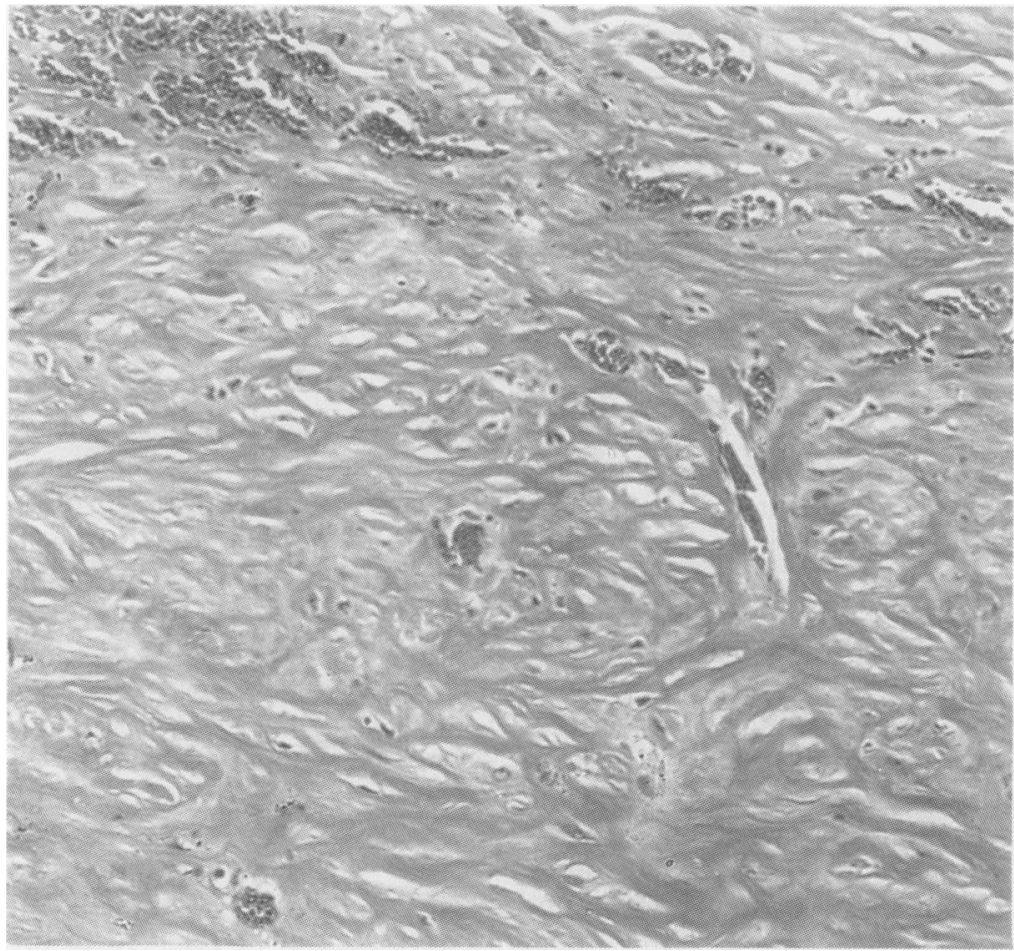

Figure 1 Storiform pattern in a desmoplastic mesothelioma: note lack of nuclei (haematoxylin and eosin).

\begin{abstract}
Results
GROSS PATHOLOGY

In $13(72 \cdot 2 \%)$ cases tumour was in the right pleural cavity. In two cases pleural tumours were bilateral, the other case being left sided. The site of one tumour was unknown. Tumour invariably encompassed the pleural cavity with extension along the interlobar fissures, infiltrating the underlying lung parenchyma and affecting the mediastinal structures including the pericardium. Spread to the chest wall was
\end{abstract}

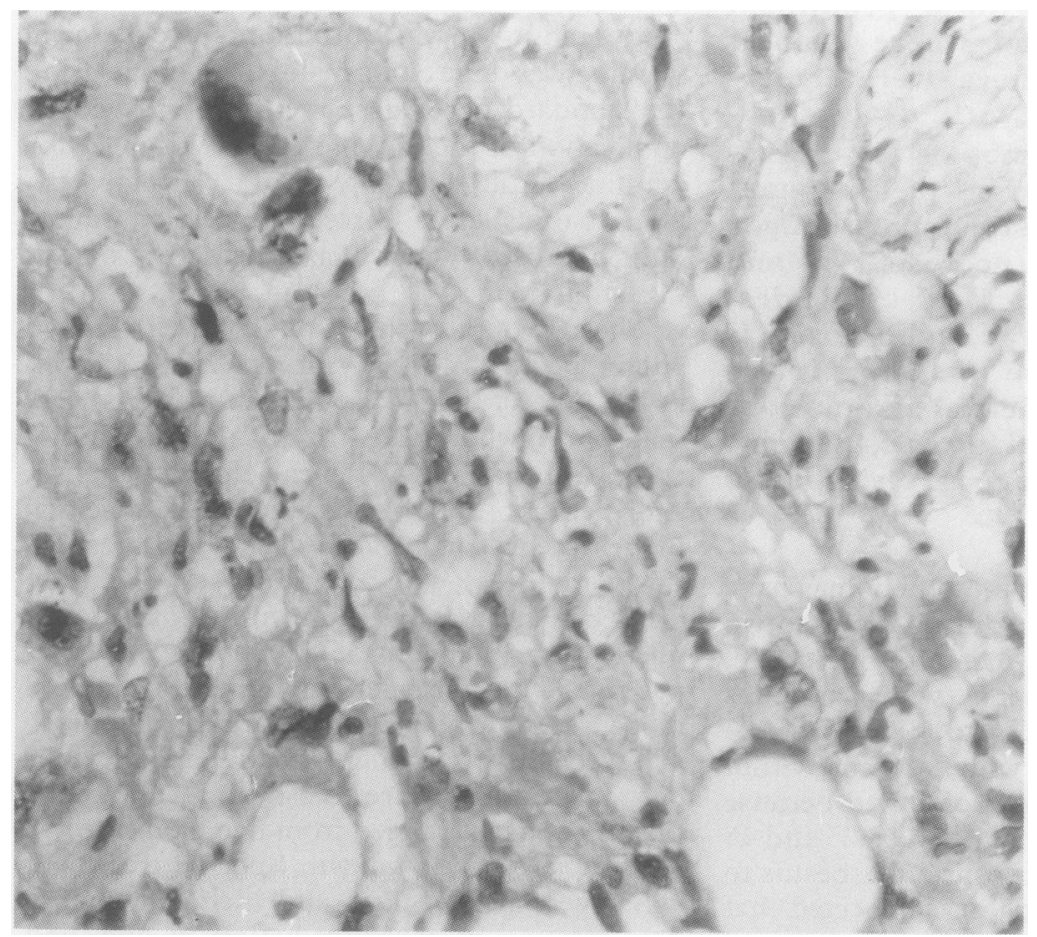

Figure 2 Nuclear hyperchromatism with tumour extending into adipose tissue. also common. The tumour varied from $0.5 \mathrm{~cm}$ to many centimetres in thickness. The underlying lung was often atelectatic.

In the 16 post mortem cases $14(87.5 \%)$ showed evidence of metastatic disease. The contralateral lung was most commonly affected ( $75 \%$ of cases). Other affected organs included liver $(n=4)$, thyroid $(n=3)$, kidney $(n=4)$, adrenal $(n=3)$, myocardium $(n=2)$, and bone $(n=3)$. Two patients had associated peritoneal tumour deposits.

\section{MICROSCOPIC PATHOLOGY}

In $17(6.6 \%)$ of 255 cases of mesothelioma reviewed there was hypocellular or sometimes acellular collagenous tissue, comprising more than $50 \%$ of tumour bulk. In 11 of the 17 $(64.7 \%)$ patients, the associated cellular areas were sarcomatous. In the remainder $(35.3 \%)$ both sarcomatous and epithelial components were present. The arrangement of the collagenous areas varied. Most commonly there was a complex network of anastomosing bands of often hyalinised collagen with a prominent storiform pattern (fig 1). This was the predominant appearance in $10(58.8 \%)$ cases. In six $(35 \cdot 2 \%)$ tumours the storiform pattern was present in combination with more spindled areas, the latter with features similar to the basket weave pattern seen in benign fibrous pleural plaques. These spindled areas, however, lacked the uniform pattern seen in a pleural plaque. Where cellular detail was assessable, there was often nuclear hyperchromatism, enlargement, and irregularity indicative of neoplasia (fig 2). In one case the spindled component was predominant. Cellular and desmoplastic areas often merged imperceptibly.

In 12 of $17(70.5 \%)$ cases collagen necrosis was present within desmoplastic areas (fig 3). In eight of $12(66.6 \%)$ cases, necrosis was prominent and easily identified. In four cases it was focal. It was always bland without associated inflammation and in haematoxylin and eosin stained sections resembled fibrinoid necrosis. Mitoses were rare, with not more than one mitosis per 10 high power fields in desmoplastic areas. Tumour infiltration of lung parenchyma was usually seen together with vascular invasion. Away from tumour, the encased lung had varied features including bronchopneumonia, thrombi, and intraalveolar oedema. In four cases early asbestos lesions in the form of fibrosis in respiratory bronchiolar walls and subpleural fibrosis were seen. There were no cases of frank asbestosis as assessed by the presence of interstitial fibrosis and many asbestos bodies. In all but one case cells within the desmoplastic component were positive for low molecular weight cytokeratin CAM $5 \cdot 2$. In nine $(52 \cdot 9 \%)$ cases more than half the cells were positive.

Asbestos fibres were counted in 14 of the 17 cases. In three lung tissue was unavailable for analysis. In 12 of $14(85.7 \%)$ cases the asbestos fibre count by light microscopy was more than 50,000 per gram of dried lung tissue indicating previous asbestos exposure (table). 


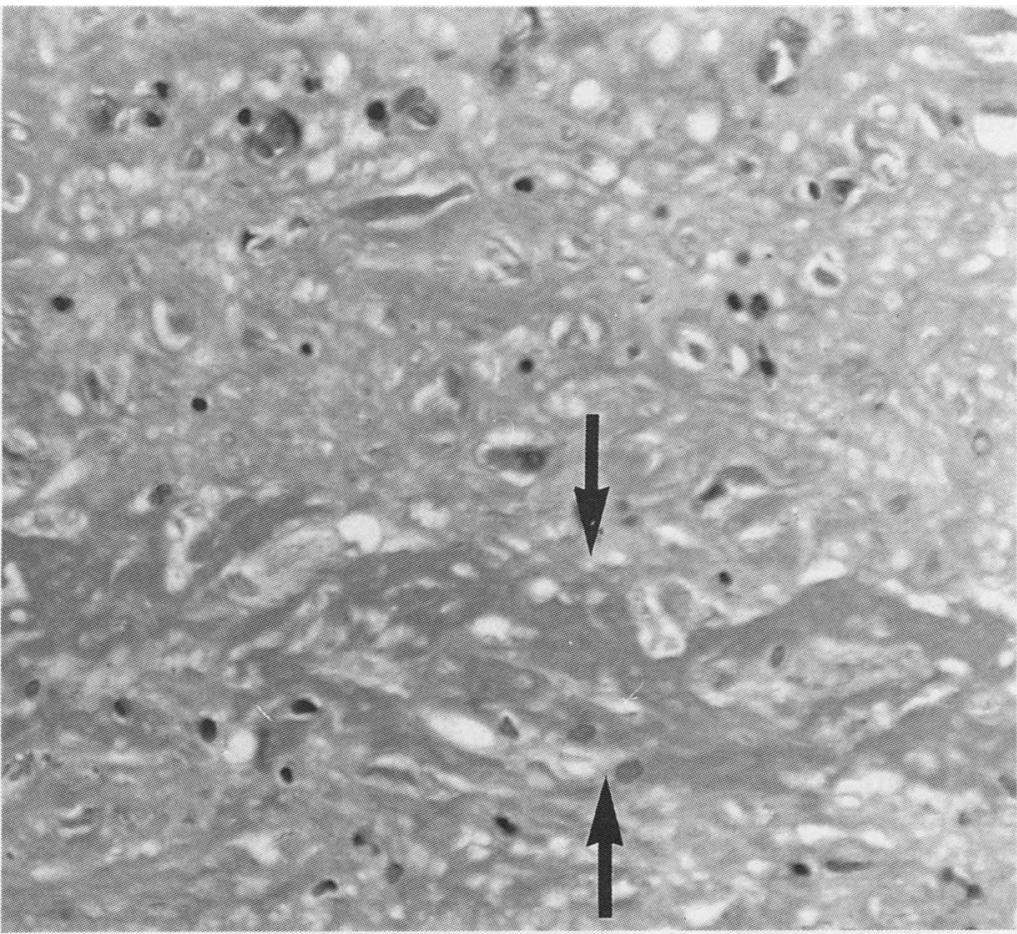

Figure 3 Collagen necrosis shown between arrows (haematoxylin and eosin).

\section{Discussion}

Malignant mesotheliomas are divided into three histological subtypes: epithelial, sarcomatous, and mixed or biphasic. These patterns are found in roughly $55 \%, 15 \%$, and $30 \%$ of cases. ${ }^{3410}$ In general terms the behaviour of epithelial mesotheliomas is similar to that of carcinomas with local spread, large pleural effusions, and metastases to regional lymph nodes. Sarcomatous mesotheliomas are associated more frequntly with distant metastases, little or no effusion, and shorter survival. Mixed mesotheliomas have intermediate features. ${ }^{11}$ Among the biphasic and sarcomatous subtypes is a group of tumours in which more than $50 \%$ of the tumour consists of dense, hypocellular collagenous tissue. These tumours have been termed desmoplastic malignant mesothelioma (DMM) ${ }^{67}$ In these studies, and in our own, there was a predominance of sarcomatous mesotheliomas (sarcomatous DMM). Unlike Cantin, ${ }^{6}$ we found no real difference in survival between cases of sarcomatous DMM (5.8 months) and biphasic DMM (6.8 months). Hillerdal, ${ }^{12}$ in a review of 4710 published cases of mesothelioma, found the survival in sarcomatous and biphasic types to be five and 11 months, respectively. The prognosis of our biphasic group with desmoplasia was similar to that of sarcomatous mesotheliomas.

Asbestos fibre counts

No of asbestos fibres per gram

of dried lung tissue

(average from two lobes)

No of cases $/ 14$

20,000

$20,000-50,000$

$50,000-100,000$

$100,000-1 \times 10 / 6$

$1 \times 10 / 6$

$1(7 \cdot 1 \%)$
Metastases were present in $87.5 \%$ of cases. These were frequently within the contralateral lung, but metastases were common at more distant sites. Cantin ${ }^{6}$ found metastases in $60 \cdot 1 \%$ of DMM. Kannerstein ${ }^{7}$ also found a higher incidence of metastases in DMM than in their general series of diffuse mesotheliomas. The survival time and the incidence of metastases, often at distant sites, strongly suggests that it is the sarcomatous component which dictates outcome.

In all tumours the desmoplastic areas consisted predominantly or in part of dense bundles of hyalinised collagen arranged in interweaving bundles, whorls, or a storiform pattern. The irregular arrangement of the collagenous component is of diagnostic importance and was present to some extent even in spindled areas which appeared superficially similar to the benign pleural plaque.

Collagen necrosis is a further diagnostic clue ${ }^{4613}$ and was found in nearly $75 \%$ of cases. It was bland with no associated inflammation and often had a confluent geographical distribution. If cytological atypia, in the form of nuclear enlargement and hyperchromatism, is seen a diagnosis of malignancy is strongly suggested. Invasion of lung parenchyma, adipose tissue, or chest wall confirms the diagnosis. Because mitoses are only occasionally present in hypocellular areas, their absence does not preclude malignancy.

The differential diagnosis of DMM includes non-specific reactive pleural fibrosis, as seen in some cases of tuberculosis, pleurisy, and rheumatoid disease. ${ }^{14}$ Clinical history may be helpful. Diffuse pleural fibrosis, ${ }^{14-16}$ associated with exposure to asbestos, has a basket weave pattern with little inflammation and no cellular fibroblastic proliferation. In all cases described by Stephens et $a l^{14}$ there was dense subpleural parenchymal interstitial fibrosis and asbestos fibre counts were very high. Localised pleural plaques should not cause diagnostic difficulty as the basket weave arrangement is regular and storiform or whorled areas are absent. Solitary fibrous tumours of the pleura (fibrous mesothelioma) are unrelated to asbestos exposure and are usually well circumscribed. ${ }^{47-20}$ They are composed of spindled cells resembling fibroblasts and arranged in a haphazard, sometimes storiform, pattern. Foci of necrosis may be found, especially in larger tumours. The tumour cells are strongly positive for vimentin and negative for keratin. This contrasts with malignant mesothelioma where the tumour cells are usually cytokeratin positive. ${ }^{212}$ Though fibrous tumours of the pleura resemble the desmoplastic areas of DMM, the macroscopic appearances and absence of keratin should indicate the correct diagnosis of fibrous tumour where this is considered. Primary sarcomas of the pleural cavity are rare, and with occasional exceptions, keratin negative. $^{23} 24$

Unfortunately, immunohistochemistry is of no assistance in the more difficult diagnostic problem of distinguishing sarcomatous mesotheliomas and DMM from pleural reactions characterised by abundant granulation 
tissue and fibrosis. ${ }^{25}$ This is because in reactive states submesothelial spindle cells acquire cytokeratin intermediate filaments, usually of low molecular weight, while retaining their connective tissue markers and so stain for both cytokeratin and vimentin. ${ }^{132526}$ The same pattern of staining is seen in most sarcomatoid mesotheliomas.

In inflammatory pleural lesions there is usually active granulation tissue formation with an acute and chronic inflammatory infiltrate and fibrosis. The fibrosis does not have a storiform or basket weave appearance and nuclear hyperchromatism is not a feature.

Apart from malignant mesothelioma, other epithelial and mesenchymal tumours may contain desmoplastic areas and, in these, there is good evidence to indicate that this is the consequence of stimulation of stromal myofibroblasts. ${ }^{27}{ }^{28}$ Many cells within the desmoplastic areas of DMM are, however, positive for cytokeratin, findings which are in accordance with those of Cantin. ${ }^{6}$ Malignant mesothelial cells, in certain cases, seem to assume the functional capacity of fibroblasts and at least one study has shown that mesothelial cells can produce collagen in tissue culture. $^{29}$

1 Spirtas R, Beebe GW, Connelly RR, et al. Recent trends in mesothelioma incidence in the United States. Am J Ind Med 1986;9:397-407.

2 Craighead JE. Current pathogenic concepts of diffuse malignant mesothelioma. Hum Pathol 1987; 18:544-57.

3 Whitwell F, Rawcliffe RM. Diffuse malignant pleural mesothelioma and asbestos exposure. Thorax 1971;26: 6-22.

4 Roggli VL, Kolbeck J, Sanfilippo F, Shelburne JD. Pathology of human mesothelioma. Aetiologic and diagnostic

5 Jones JSP. Asbestos fibres and their biological effects on the mesothelium. In: Jones JSP, ed. Pathology of the mesomesothelium. In: Jones JSP, ed. Pathology of

6 Cantin R, Al-Jabi M, McCaughey WTE. Desmoplastic diffuse mesothelioma. Am J Surg Pathol 1982;6:215-22.

7 Kannerstein M, Churg J. Desmoplastic diffuse malignan mesothelioma. In: Fenoglio CM, Wolff M, eds. Progress in surgical pathology. Vol II. New York: Masson Publishing 1980:19-29.

8 Hasleton PS, Kelehan P, Whittaker JS, Burslem RW, Turner L. Benign and malignant struma ovarii. Arch Pathol Lab Med 1978;102:180-4.

9 Ashcroft T, Heppleston AG. The optical and electron microscopic determination of pulmonary asbestos fibre concentration and its relation to the human pathological reaction. J Clin Pathol 1973;26:224-34.

10 Chailleux E, Dabouis G, Pioche D, et al. Prognostic factors in diffuse malignant pleural mesothelioma. A study of 167 patients. Chest 1988;93:159-62.

11 Law MR, Hodson ME, Heard BE. Malignant mesothelioma of the pleura: relation between histological type and clinical behaviour. Thorax 1982;37:810-5.

12 Hillerdal G. Malignant mesothelioma 1982: Review of 4710 published cases. Br J Dis Chest 1983;77:321-43.

13 Bolen JW, Hammar SP, McNutt MA. Reactive and neoplastic serosal tissue. A light microscopic, ultrastructural and tic serosal tissue. A light microscopic, ultrastructural and immunocy

14 Stephens M, Gibbs AR, Pooley FD, Wagner JC. Asbestos induced diffuse pleural fibrosis: pathology and $\mathrm{min}$ eralogy. Thorax 1987;42:583-8.

15 Albelda SM, Epstein DM, Gefter WB, Miller WT. Pleura thickening: its significance and relationship to asbesto dust exposure. Am Rev Respir Dis 1982;126:621-4.

16 McLoud TC, Woods BO, Carrington CB, Eplet GR, Gaensler EA. Diffuse pleural thickening in an asbestosexposed population. Am J Radiol 1985;144:9-18.

17 Carter D, Otis CN. Three types of spindle cell tumours of the pleura. Fibroma, sarcoma and sarcomatoid mesothelioma. Am J Surg Pathol 1988;12:747-53.

18 Dervan PA, Tobin B, O'Connor M. Solitary (localised) fibrous mesothelioma: evidence against mesothelial cel forous mesothelioma: evidence again

19 Said JW, Nash G, Banks-Schlegel S, Sassoon AF, Shintaku PI. Localised fibrous mesothelioma. An immunohistochemical and electron microscopic study. Hum Patho 1984;15:440-3.

20 Briselli M, Mark EJ, Dickersin R. Solitary fibrous tumours of the pleura: Eight new cases and review of 360 cases in the literature. Cancer 1981;47:2678-89.

21 Corson JM, Pinkus GS. Mesothelioma: Profile of keratin proteins and carcinoembryonic antigen. An immunoperoxidase study of 20 cases and comparison with pulmonary adenocarcinomas. Am J Pathol 1982;108:80-7.

22 Montag AG, Pinkus GS, Corson JM. Keratin protei immunoreactivity of sarcomatoid and mixed types of diffuse malignant mesothelioma. An immunoperoxidase diffuse malignant mesothelioma. An immuno

23 Corson JM, Weiss LM, Banks-Schlegel SP, Pinkus G Keratin proteins in synovial sarcoma. Am J Surg Patho 1983;7:107-9.

24 Chase DR, Weiss SW, Enzinger FM, Langloss JM. Keratin in epithelioid sarcoma. An immunohistochemical study. Am J Surg Pathol 1984;8:435-41.

25 Al-Izzi M, Thurlow NP, Corrin B. Pleural mesothelioma of connective tissue type, localised fibrous tumour of pleura and reactive submesothelial hyperplasia. An immunohistochemical comparison. J Pathol 1989;158:41-4.

26 Epstein JI, Budin RE. Keratin and epithelial membrane antigen immunoreactivity in non-neoplastic fibrous antigen immunoreactivity in non-neoplastic fibrous pleural lesions. Implications for the diagnosis of

27 Seemayer TA, Lagace R, Schurch W, Tremblay G. Myofibroblasts in the stroma of invasive and metastatic carcinoma. Am J Surg Pathol 1979;3:525-33.

28 Lagace R, Schurch W, Seemayer TA. Myofibroblasts in sof tissue sarcomas. Virchows Arch (Pathol Anat) 1980;389:1-11.

29 Raftery AT. Regeneration of parietal and visceral peritoneum: an electron microscopical study. J Anat 1973; 115:375-92. 